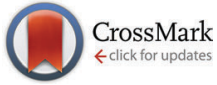

Cite this: J. Mater. Chem. C, 2016 , 4, 4756

Received 1st March 2016 Accepted 13th April 2016 DOI: $10.1039 / c 6 t c 00893 c$

www.rsc.org/MaterialsC

\title{
Colloidal $\mathrm{AgSbSe}_{2}$ nanocrystals: surface analysis, electronic doping and processing into thermoelectric nanomaterials $\dagger$
}

\author{
Yu Liu, ${ }^{a}$ Doris Cadavid, ${ }^{\star a}$ Maria Ibáñez, ${ }^{\text {bc }}$ Jonathan De Roo, ${ }^{d}$ Silvia Ortega, ${ }^{a}$ \\ Oleksandr Dobrozhan, ${ }^{a}$ Maksym V. Kovalenko ${ }^{\text {bc }}$ and Andreu Cabot*ae
}

\begin{abstract}
We present a high-yield and scalable colloidal synthesis to produce monodisperse $\mathrm{AgSbSe}_{2}$ nanocrystals (NCs). Using nuclear magnetic resonance (NMR) spectroscopy, we characterized the NC surface chemistry and demonstrate the presence of surfactants in dynamic exchange, which controls the NC growth mechanism. In addition, these NCs were electronically doped by introducing small amounts of bismuth. To demonstrate the technological potential of such processed material, after ligand removal by means of $\mathrm{NaNH}_{2}, \mathrm{AgSbSe}_{2} \mathrm{NCs}$ were used as building blocks to produce thermoelectric (TE) nanomaterials. A preliminary optimization of the doping concentration resulted in a thermoelectric figure of merit ( $Z T)$ of 1.1 at $640 \mathrm{~K}$, which is comparable to the best $Z T$ values obtained with a $\mathrm{Pb}$ - and Te-free material in this middle temperature range, with the additional advantage of the high versatility and low cost associated with solution processing technologies.
\end{abstract}

\section{Introduction}

I-V-VI 2 semiconductors, particularly AgSbSe ${ }_{2}$, show very attractive and yet to be exploited functional properties. AgSbSe ${ }_{2}$, with a rock-salt crystal structure, is highly suitable, for instance, as a phase change recording material in rewritable optical memories due to drastic resistance drop upon crystallization from an amorphous state. ${ }^{1-6}$ Within the AgSbSe 2 structure, Ag and $\mathrm{Sb}$ are generally assumed to randomly occupy the Na sites of the NaCl-type lattice, ${ }^{7-10}$ which is consistent with $a b$ initio calculations showing the total energies of the various ordered structures to be very close, thus allowing the presence of a significant density of disorder defects and mixed phases. A careful structural characterization has shown domains of ordering, not only in $\mathrm{AgSbSe}_{2},{ }^{11}$ but also in the related $\mathrm{AgSbTe}_{2},{ }^{12}$ correlating well with calculations showing a rhombohedral

\footnotetext{
${ }^{a}$ Catalonia Institute for Energy Research-IREC, Jardins de les Dones de Negre 1, 08930, Sant Adrià del Besos, Spain.E-mail: dcadavid@irec.cat, acabot@irec.cat

${ }^{b}$ Institute of Inorganic Chemistry, Department of Chemistry and Applied Biosciences, ETH Zürich, CH-8093, Switzerland

${ }^{c}$ Empa-Swiss Federal Laboratories for Materials Science and Technology, Dübendorf, CH-8600, Switzerland

${ }^{d}$ Ghent University, Department of Inorganic and Physical Chemistry, Krijgslaan 281 S3, 9000 Gent, Belgium

${ }^{e}$ Institució Catalana de Recerca i Estudis Avançats - ICREA, 08010 Barcelona, Spain

$\dagger$ Electronic supplementary information (ESI) available: Additional experimental details and TEM, XRD, EDX, and NMR data. See DOI: 10.1039/c6tc00893c
}

ordering to be the most energetically favorable in $\mathrm{AgSbSe}_{2} \cdot{ }^{13}$ Atomic ordering within the $\mathrm{AgSbSe}_{2}$ crystal has a significant impact on the electronic structure near the band gap, which dominates the electronic properties ${ }^{14}$ and provides the material with ferroelectric properties. ${ }^{11}$

$\mathrm{AgSbSe}_{2}$ is a narrow, indirect band-gap semiconductor (0.03-0.10 eV) showing p-type conductivity. ${ }^{15}$ However, an apparent optical bandgap of $0.6-1.1 \mathrm{eV}^{2,15-18}$ and a high optical absorption coefficient $\left(10^{4} \mathrm{~cm}^{-1}\right)$ have also motivated the use of $\mathrm{AgSbSe}_{2}$ for photovoltaic applications. ${ }^{2,17,19-21}$

The AgSbSe ${ }_{2}$ band structure is characterized by a multi-peak valence band maximum, which potentially results in a high effective mass for holes and thus a high Seebeck coefficient. In addition, $\mathrm{AgSbSe}_{2}$ features a strong anharmonic bonding arrangement associated with the $\mathrm{Sb} 5 \mathrm{~s}^{2}$ lone pair, which translates into strong phonon-phonon interactions that reduce the lattice thermal conductivity to values close to the amorphous limit. This intrinsically low thermal conductivity and the appropriate electronic band structure make $\mathrm{AgSbSe}_{2}$ and $\mathrm{AgSbTe}_{2}$ promising candidates for TE applications in the intermediate temperature range $(500-700 \mathrm{~K}){ }^{16,22-28}$ While $\mathrm{AgSbTe}_{2}$ exhibits higher electrical conductivity, and its nanostructured alloys with GeTe (TAGS) ${ }^{29}$ and PbTe (LAST-m) ${ }^{30}$ are well known for their remarkable $Z T$ values, $\mathrm{AgSbSe}_{2}$ is advantageous in terms of thermal stability, abundance of constituting elements and cost. Bulk AgSbSe ${ }_{2}$ is typically produced by the solid state reaction of its highly purified elements at temperatures above $1000 \mathrm{~K}$. A relatively large variety of extrinsic electronic dopants, introduced 
as pure elements in the reaction mixture, have been tested, including the substitution of $\mathrm{Sb}^{3+}$ by monovalent $\mathrm{Na}^{+},{ }^{28}$ divalent $\mathrm{Pb}^{2+},{ }^{16} \mathrm{Zn}^{2+},{ }^{23} \mathrm{Sn}^{2+},{ }^{24} \mathrm{Cd}^{2+},{ }^{26} \mathrm{Mg}^{2+},{ }^{27} \mathrm{Ba}^{2+},{ }^{27}$ and peculiarly by trivalent $\mathrm{Bi}^{3+} \cdot{ }^{16}$ Extrinsic dopants can also introduce additional disorder and point defect scattering reducing the material thermal conductivity. In some cases, e.g. $\mathrm{Na}^{+}, \mathrm{Zn}^{2+}$ and $\mathrm{Ba}^{2+}$ can also introduce nanoprecipitates with modified stoichiometry, e.g. Na-rich AgSbSe ${ }_{2}$ or even of secondary phases, e.g. ZnSe and $\mathrm{BaSe}_{3}$, which further reduce thermal conductivity and allow reaching $Z T$ values of up to $\sim 1.1 .^{23,27}$ Besides, intrinsic doping strategies based on tuning the material stoichiometry by modifying, for instance, the $\mathrm{Sb}$ content ${ }^{25}$ and reaching $Z T$ values of up to $\sim 1$ have also been tested by solid state reaction methods.

The preparation of $\mathrm{AgSbSe}_{2}$ with controlled properties by means of solution processing methods is especially appealing to investigate the structural and compositional dependent functional properties, optimize the material for relevant applications and develop high performance cost-effective products. In particular, the availability of $\mathrm{AgSbSe}_{2}$ NCs with tuned size, shape, composition and phase would allow high density data storage systems, cost-effective photovoltaic devices and high performance thermoelectric modules to be produced by highly versatile, low-cost, high-throughput and high-yield solutionbased bottom-up technologies. ${ }^{31-36}$ However, to the best of our knowledge, no synthesis protocol to produce colloidal $\mathrm{AgSbSe}_{2} \mathrm{NCs}$ is currently available.

Here, we detail the first colloidal synthesis of monodisperse $\mathrm{AgSbSe}_{2}$ NCs on the gram scale, determine the NC surface composition by NMR analysis and demonstrate the possibility of electronically doping this material with controlled amounts of bismuth. Finally, we elaborate upon a procedure to remove organic ligands and demonstrate the suitability of these NCs to produce high performance thermoelectric nanomaterials.

\section{Experimental section}

\subsection{Chemicals and solvents}

Silver nitrate $\left(\mathrm{AgNO}_{3}, \geq 99 \%\right)$, antimony(III) chloride $\left(\mathrm{SbCl}_{3}, 99 \%\right)$, selenium powder (Se, 99.5\%), bismuth(III) acetate $\left(\mathrm{Bi}\left(\mathrm{CH}_{3} \mathrm{CO}_{2}\right)_{3}\right.$, $>99.99 \%)$, sodium amide $\left(\mathrm{NaNH}_{2}, 95 \%\right)$, 1-dodecanethiol (DDT, $\geq 98 \%$ ), oleic acid (OA, 90\%), 1-octadecene (ODE, 90\%), and oleylamine (OLA, 70\%) were purchased from Sigma-Aldrich. Analytical grade acetone and chloroform were obtained from various sources. All chemicals were used as received without further purification. Syntheses were carried out using a standard vacuum/dry argon Schlenk line.

\subsection{Selenium precursor stock solution}

A $0.5 \mathrm{M}$ selenium stock solution was prepared by dissolving selenium powder $(1.5792 \mathrm{~g}, 20 \mathrm{mmol})$ in $20 \mathrm{ml}$ OLA and $20 \mathrm{ml}$ DDT at room temperature, cycled between vacuum and argon to remove the oxygen in the flask, and then stirred under an $\mathrm{Ar}$ atmosphere until Se powder was completely dissolved.

\subsection{Synthesis of $\mathrm{AgSbSe}_{2} \mathrm{NCs}$}

In a typical synthesis, $\mathrm{AgNO}_{3}(5 \mathrm{mmol}, 0.849 \mathrm{~g}), \mathrm{SbCl}_{3}(5 \mathrm{mmol}$, $1.141 \mathrm{~g}$ ), $70 \mathrm{ml}$ OLA and $10 \mathrm{ml}$ OA were mixed in a $250 \mathrm{ml}$ threeneck flask at room temperature under magnetic stirring for $30 \mathrm{~min}$ in order to remove low boiling point impurities. Afterwards, the solution was maintained at $80{ }^{\circ} \mathrm{C}$ under vacuum for $60 \mathrm{~min}$. At around $70{ }^{\circ} \mathrm{C}$ the solution became yellowish and changed from ivory yellow to yellowish-brown during the time at $80{ }^{\circ} \mathrm{C}$. Then the solution was heated to $120{ }^{\circ} \mathrm{C}$ for another 10 min under vacuum. After these steps, argon was introduced and the reaction was rapidly heated to $220^{\circ} \mathrm{C}$. At this temperature, $20 \mathrm{ml}$ of a $0.5 \mathrm{M}$ selenium solution were quickly injected. After injection, the color of the solution changed immediately from yellowish-brown to dark brown, indicating the NC nucleation. NCs were allowed to grow for $30 \mathrm{~min}$ at $220{ }^{\circ} \mathrm{C}$. Afterwards, the colloidal solution was rapidly cooled to room temperature through a water bath. The resultant dark brown solution was cleaned by centrifugation at $6000 \mathrm{rpm}$ for $5 \mathrm{~min}$ using chloroform as a solvent and acetone as a non-solvent.

\subsection{Synthesis of Bi-doped AgSbSe ${ }_{2} \mathrm{NCs}$}

Bi-doped $\mathrm{AgSbSe}_{2}$ NCs were synthesized by using the same process as the pristine NCs, but replacing the desired amount of $\mathrm{SbCl}_{3}$ by $\mathrm{Bi}\left(\mathrm{CH}_{3} \mathrm{CO}_{2}\right)_{3}$. To produce $\mathrm{AgSb}_{0.98} \mathrm{Bi}_{0.02} \mathrm{Se}_{2}$, the precursor molar ratio was $\mathrm{SbCl}_{3} / \mathrm{Bi}\left(\mathrm{CH}_{3} \mathrm{CO}_{2}\right)_{3}=0.98 / 0.02$ (4.9 mmol/0.1 mmol).

\subsection{Nuclear magnetic resonance (NMR)}

NMR measurements were recorded on a Bruker Avance III HD spectrometer operating at a ${ }^{1} \mathrm{H}$ frequency of $500.26 \mathrm{MHz}$ and equipped with a BBFO-Z probe. The sample temperature was set to $298.2 \mathrm{~K}$. One dimensional (1D) ${ }^{1} \mathrm{H}$ and $2 \mathrm{D}$ NOESY (Nuclear Overhauser Effect Spectroscopy) spectra were acquired using standard pulse sequences from the Bruker library. For the quantitative $1 \mathrm{D}{ }^{1} \mathrm{H}$ measurements, $64 \mathrm{k}$ data points were sampled with the spectral width set to $20 \mathrm{ppm}$ and a relaxation delay of $30 \mathrm{~s}$. The NOESY mixing time was set to $300 \mathrm{~ms}$ and 4096 data points in the direct dimension and 512 data points in the indirect dimension were sampled, with the spectral width set to $12 \mathrm{ppm}$. Diffusion measurements (2D DOSY) were performed using a double stimulated echo sequence for convection compensation and with monopolar gradient pulses. ${ }^{37}$ Smoothed rectangle gradient pulse shapes were used throughout. The gradient strength was varied quadratically from 2-95\% of the probe's maximum value in 64 increments, with the gradient pulse duration and diffusion delay optimized to ensure a final attenuation of the signal in the final increment of less than $10 \%$ relative to the first increment. For $2 \mathrm{D}$ processing, the spectra were zero filled until a 4096-2048 real data matrix. Before Fourier transformation, the 2D spectra were multiplied with a squared cosine bell function in both dimensions, and the 1D spectra were multiplied with an exponential window function. Concentrations were obtained using the Digital ERETIC method, as provided in the standard software of Bruker. The diffusion coefficients were obtained by fitting 
the appropriate Stejskal-Tanner equation to the signal intensity decay. $^{38}$

\subsection{NC ligand displacement (LD)}

$\mathrm{NaNH}_{2}$ was used to displace organic ligands from the AgSbSe and $\mathrm{AgSb}_{0.98} \mathrm{Bi}_{0.02} \mathrm{Se}_{2} \mathrm{NC}$ surface. In a typical procedure, $\mathrm{AgSbSe}_{2} \mathrm{NCs}(500 \mathrm{mg}$ ) were dispersed in $15 \mathrm{ml}$ of chloroform and mixed at room temperature with $5 \mathrm{ml}$ of $\mathrm{NaNH}_{2}$ solution $(0.02 \mathrm{M})$ in methanol. The solution was shaken for 1-2 min to displace the organic ligands attached to the NC surface. This process was repeated twice. Afterward, NCs were precipitated by centrifugation and they were thoroughly purified using chloroform and acetone to remove the remaining organic surfactants and excess $\mathrm{NaNH}_{2}$. Finally, AgSbSe 2 NCs were precipitated and dried under vacuum to obtain a fine nanopowder.

\subsection{Bulk nanomaterials}

$\mathrm{AgSbSe}_{2}$ and $\mathrm{AgSb}_{0.98} \mathrm{Bi}_{0.02} \mathrm{Se}_{2}$ nanopowders obtained from drying $15 \mathrm{~nm}$ NCs were loaded into graphite dies and compacted into pellets $(\varnothing 10 \mathrm{~mm} \times \sim 1.5 \mathrm{~mm})$ in an Ar atmosphere using a custom-made hot press. The process temperature was maintained between $350{ }^{\circ} \mathrm{C}$ and $360{ }^{\circ} \mathrm{C}$ for $30 \mathrm{~min}$ and the pressure was set to $70 \mathrm{MPa}$. In this system, heat is provided by an induction coil operated in the RF range applied directly to a graphite die, which acts as a susceptor, resulting in a heating ramp of about $20{ }^{\circ} \mathrm{C}$ per second. The density of the pressed pellets was always higher than $93 \%$ of their theoretical value.

\subsection{Structural and chemical characterization}

X-ray diffraction analyses were carried out on a Bruker AXS D8 ADVANCE X-ray diffractometer using $\mathrm{Cu}-\mathrm{K} \alpha$ radiation $(\lambda=0.15406 \AA)$. The size and shape of initial NCs were examined by transmission electron microscopy (TEM) using a ZEISS LIBRA 120, operating at $120 \mathrm{kV}$. High resolution TEM (HRTEM) images were recorded using a JEOL JEM-2200FS microscope operated at $200 \mathrm{kV}$. For TEM analysis colloidal nanoparticles in chloroform were supported by drop-casting the solution on a carbon-coated copper grid at room temperature and ambient atmosphere. For HRTEM characterization of the pellets, we manually ground a piece of the measured pellet and mixed the obtained powder with TCE (tetrachloroethylene). The powder suspension was drop casted onto a carbon coated copper grid at room temperature and ambient atmosphere. Field-emission scanning electron microscopy (SEM) was carried out on an Auriga Zeiss at $5.0 \mathrm{kV}$. Quantitative elemental analysis was made by means of an energy dispersive X-ray spectroscopy (EDX) detector attached to a SEM.

\subsection{Thermoelectric measurements}

Seebeck coefficient was measured by using a static DC method. Electrical resistivity data were obtained by a standard fourprobe method. Both the Seebeck coefficient and the electrical resistivity were measured simultaneously in a LSR-3 LINSEIS system in the range between room temperature and $700 \mathrm{~K}$, under a helium atmosphere. XFA 600 xenon flash equipment was used to determine the thermal diffusivities of the samples.
The carrier concentration and mobility were measured using the Hall measurement system (PPMS-9T, Quantum Design Inc., USA) at room temperature under a magnetic field of $2 \mathrm{~T}$. The thermal conductivity was calculated using $\kappa=\lambda C_{\mathrm{p}} \rho$, where $\lambda$ is the thermal diffusivity, $C_{\mathrm{p}}$ is the heat capacity, and $\rho$ is the mass density of the specimen. The specific heat capacity $\left(C_{\mathrm{p}}\right)$ was measured by means of a differential scanning calorimetry method (DSC model T2000, TA Instruments), and the density $(\rho)$ values used here were calculated using Archimedes' method.

\section{Results and discussion}

Fig. 1a shows a representative TEM micrograph of the spherical $15 \pm 1 \mathrm{~nm} \mathrm{AgSbSe}{ }_{2}$ NCs obtained following the above procedure. NCs could be dispersed in non-polar solvents such as toluene, hexane or chloroform to form a stable, dark brown dispersion (Fig. 1b). XRD analysis showed AgSbSe ${ }_{2}$ NCs to have a cubic structure with a $F m \overline{3} m$ space group $(a=5.78 \AA$ ) (Fig. 1d, JCPDS card No. 00-012-0379). No order between the Ag and Sb ions could be discerned from the XRD patterns, which is consistent with the similar scattering factors of $\mathrm{Ag}$ and $\mathrm{Sb}$ ions. Importantly, no secondary phases were detected in the XRD pattern, pointing towards the phase purity of the synthesized NCs. EDX analysis showed the composition of silver, antimony, and selenium to be consistent with stoichiometric $\mathrm{AgSbSe}_{2}$, within the experimental error of $\sim 2 \%$ (Fig. S1, ESI $\dagger$ ).

The presence of OLA and OA in the precursor solution was required to dissolve $\mathrm{AgNO}_{3}$ and $\mathrm{SbCl}_{3}$, respectively. ${ }^{39}$ The absence of $\mathrm{OA}$ prevented the incorporation of $\mathrm{Sb}$ and thus

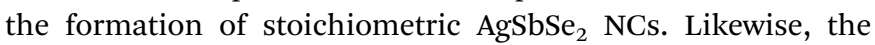
absence of OLA resulted in a polydisperse mixture of $\mathrm{AgSbSe}_{2}$ and $\mathrm{Ag}_{2} \mathrm{Se}$ (Fig. S2 and $\mathrm{S} 3$, ESI $\dagger$ ).

Although the reaction mixture already turned dark brown upon injection of the selenium precursor, relatively long reaction times were necessary to produce monodisperse NCs (Fig. 2 and Fig. S4, ESI $\dagger$ ). Within the first $15 \mathrm{~min}$, NCs with a bimodal size distribution were obtained. This bimodal distribution possibly originates from an extended nucleation time due to a relatively low reactivity of the precursor complexes, which
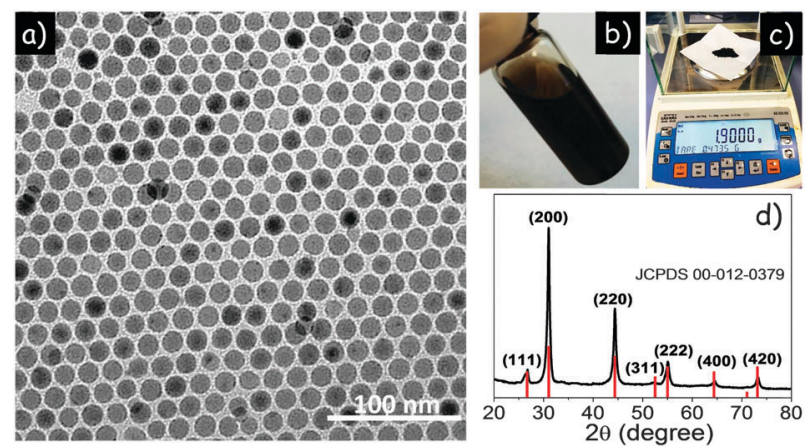

Fig. 1 (a) TEM micrograph of the AgSbSe 2 NCs. (b) NCs dispersed in chloroform. (c) The amount of NCs produced per batch. (d) The XRD pattern of $\mathrm{AgSbSe}_{2}$. 

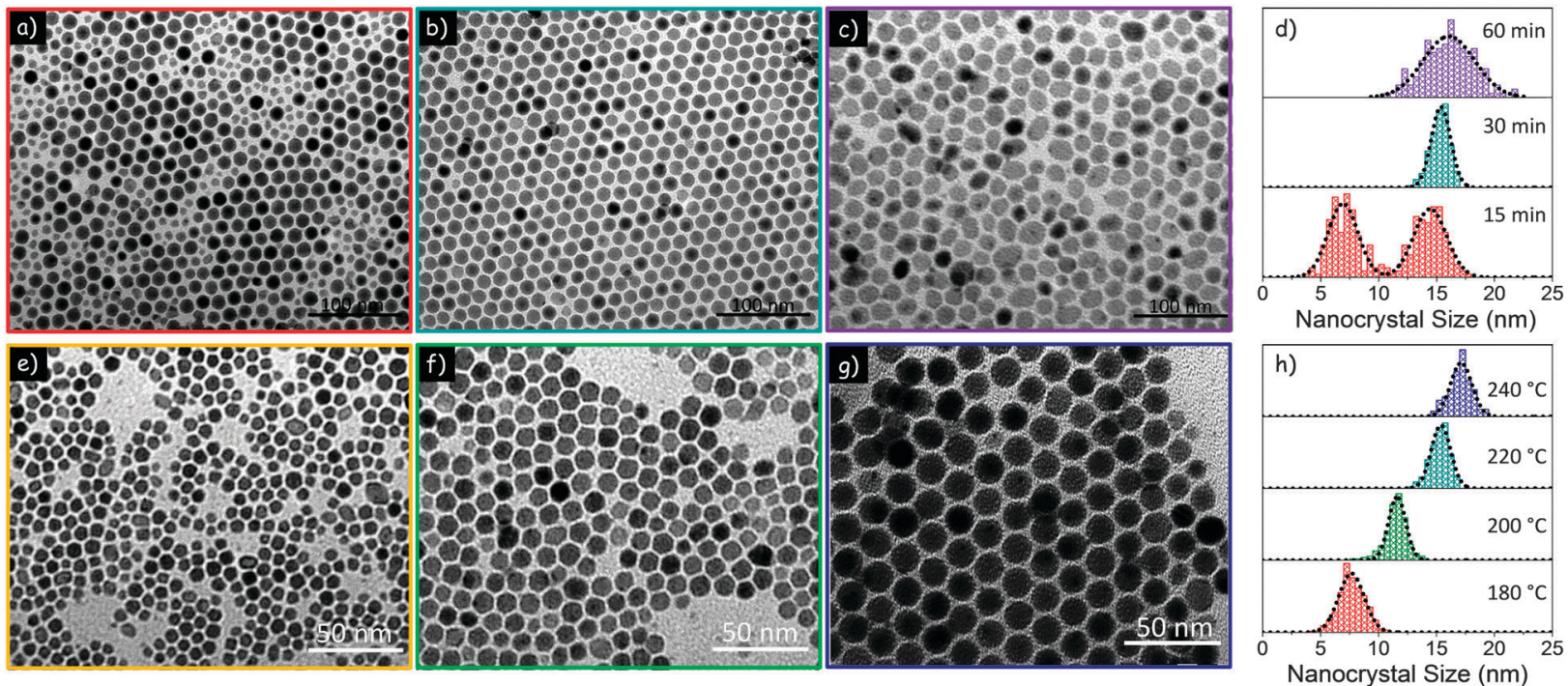

Fig. 2 (a-c) TEM micrographs of $\mathrm{AgSbSe}_{2} \mathrm{NCs}$ prepared at a fixed temperature, $220{ }^{\circ} \mathrm{C}$, and different reaction times: (a) 15 min; (b) 30 min; and (c) $60 \mathrm{~min}$. (d) Histogram of the particle size distribution for NCs produced at increasing reaction times. (e and f) TEM micrographs of AgSbSe 2 NCs prepared at different reaction temperatures and a fixed reaction time, $30 \mathrm{~min}$ : (e) $180{ }^{\circ} \mathrm{C}$; (f) $200{ }^{\circ} \mathrm{C}$; and (g) $240{ }^{\circ} \mathrm{C}$ (5 min). (h) Histogram of the measured particle size distribution for NCs produced at increasing reaction temperatures.

translates into a slow NC growth that prevents a rapid monomer reduction in the supersaturation. ${ }^{40} \mathrm{~A}$ focusing of the size distribution, most probably mediated by an Ostwald ripening process, was observed up to $30 \mathrm{~min}$ of reaction time, when the narrowest size distribution was obtained. Larger reaction times resulted in a non-uniform growth of the NCs, increasing the size distribution width.

Consistent with this growth mechanism, an increase of the injection and reaction temperature and thus of the monomer reactivity resulted in larger NCs. Therefore, fixing the reaction time, the $\mathrm{AgSbSe}_{2} \mathrm{NC}$ size could be easily tailored in the range from $7 \pm 1 \mathrm{~nm}$ to around $17 \pm 2 \mathrm{~nm}$ by just adjusting the reaction temperature in the range from 180 to $240{ }^{\circ} \mathrm{C}$ (Fig. 2 and Fig. S5, ESI $\dagger$ ). Reaction temperatures below $180{ }^{\circ} \mathrm{C}$ did not allow the growth of $\mathrm{AgSbSe}_{2}$ NCs. Higher reaction temperatures resulted in highly polydisperse NCs.

In the ${ }^{1} \mathrm{H}$ nuclear magnetic resonance (NMR) spectrum of the resulting $\mathrm{NC}$ dispersion in deuteroform, slightly broadened resonances are observed which correspond to an oleyl chain (Fig. 3a). Since the integrations from the alkene resonance (5) and the $\mathrm{CH}_{3}$ resonance (6) fit the expected 2:3 ratio we conclude that fully saturated molecules are not present. However, it is difficult to distinguish OA from OLA since the characteristic $\mathrm{CH}_{2}$ resonances (1 and 2) next to the functional group are not detected, presumably due to the interaction of the ligand with the NC surface. The nuclear Overhauser effect spectrum (NOESY) (Fig. 3b) indeed confirms that the oleyl species interact with the surface since negative (black) nOe cross peaks are observed. ${ }^{41}$ The total ligand concentration was determined to be $4.5 \mathrm{mM}$ (based on resonance 6) and with the NC concentration and NC size, a rather low ligand density of $1.2 \mathrm{~nm}^{-2}$ was calculated. The diffusion ordered NMR spectrum (DOSY, Fig. 3c) features two sets of resonances (apart from acetone), with a different diffusion coefficient, $D$. This indicates that two species are present which diffuse with a different speed. However, none of the two species diffuses slow enough
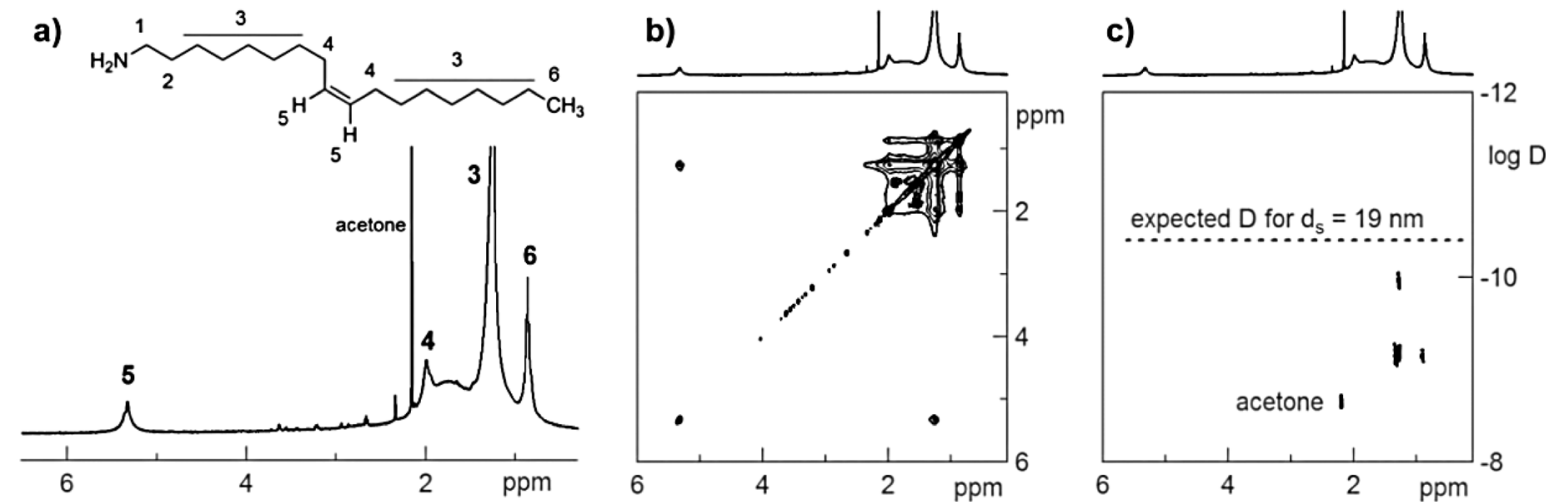

Fig. 3 (a) ${ }^{1} \mathrm{H}$ NMR spectrum of $\mathrm{AgSbSe}_{2} 15 \pm 2 \mathrm{~nm} \mathrm{NCs}(4 \mu \mathrm{M})$ and the corresponding (b) NOESY and (c) DOSY spectra. 
to correspond to a $15 \mathrm{~nm}$ nanocrystal plus a tightly bound ligand shell, which would yield a solvodynamic diameter, $d_{\mathrm{s}}=19 \mathrm{~nm}$. We thus infer that the ligands are not tightly bound but are in a dynamic exchange regime between a free state and a bound state. Therefore, the ligand density that was calculated earlier is a maximum value and, in reality, the ligands only spend part of their time on the surface. A low bond strength of the ligands to the NC surface and the observed ligand dynamic exchange are consistent with the NC growth not being limited by the surfactant concentration, but by the growth kinetics.

To elucidate the nature of the two species in DOSY, we stripped the ligands from the NC surface by the addition of TFA. After removal of the NCs by centrifugation, the supernatant was again measured using NMR (Fig. S6, ESI $\dagger$ ) and the characteristic resonances of both OA and OLA were observed with a relative abundance of $18 \%$ and $82 \%$ respectively.

Controlled amounts of bismuth were incorporated within the AgSbSe ${ }_{2}$ NCs by replacing a small amount of $\mathrm{SbCl}_{3}$ by an equivalent amount of $\mathrm{Bi}\left(\mathrm{CH}_{3} \mathrm{COO}\right)_{3}$ in the precursor solution. At relatively low $\mathrm{Bi}$ concentrations, $[\mathrm{Bi}]<5 \%$, EDX analysis showed the final Bi composition in the NCs to match, within the experimental error, the nominal concentration of introduced $\mathrm{Bi}\left(\mathrm{CH}_{3} \mathrm{COO}\right)_{3}$. The incorporation of $\mathrm{Bi}$ ions within the $\mathrm{AgSbSe}_{2}$ lattice was confirmed by a slight peak shift toward lower angles in the XRD pattern (Fig. 4b), pointing towards an increase of the lattice parameters. Bi-doping had also an evident influence on the size and shape distribution of the produced NCs (Fig. 4a).

To use $\mathrm{AgSbSe}_{2}$ NCs in solid-state devices that require an efficient transport of charge carriers, original insulating organic ligands have to be removed. Among the different ligand displacement agents tested, $\mathrm{NaNH}_{2}$ was the most effective one, as characterized by Fourier-transform infrared spectroscopy (FTIR). After ligand removal with a $0.02 \mathrm{M} \mathrm{NaNH}_{2}$ solution, $\mathrm{AgSbSe}_{2}$ could not be re-dispersed in non-polar solvents and the strong $\mathrm{C}-\mathrm{H}$ vibration modes $\left(2850-3000 \mathrm{~cm}^{-1}\right)$ and the bands corresponding to $\mathrm{C}-\mathrm{C}, \mathrm{C}-\mathrm{N}$ and $\mathrm{NH}_{2}\left(700-1650 \mathrm{~cm}^{-1}\right)$ had completely disappeared from the FTIR spectrum (Fig. 5), proving the effective removal of OLA and OA from the NC surface.

After ligand displacement, dried $15 \mathrm{~nm} \mathrm{AgSbSe}{ }_{2}$ NCs were hot-pressed into $10 \mathrm{~mm}$ diameter and $1.5 \mathrm{~mm}$ thick pellets at $350{ }^{\circ} \mathrm{C}$ under $70 \mathrm{MPa}$ pressure for $30 \mathrm{~min}$. All pellets produced
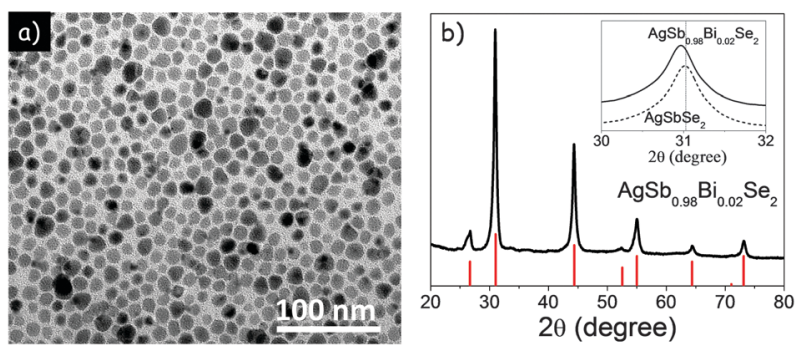

Fig. 4 (a) TEM micrograph of $\mathrm{AgSb}_{0.98} \mathrm{Bi}_{0.02} \mathrm{Se}_{2} \mathrm{NCs}$ and (b) their XRD pattern including the reference JCPDS 00-012-0379. The inset shows details of the (200) peak.

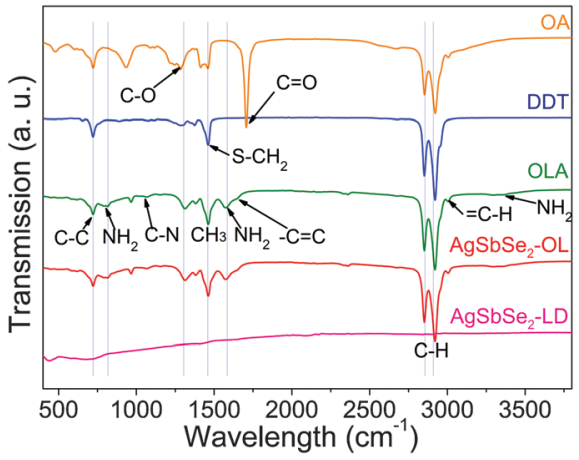

Fig. 5 From top to down, FTIR spectra of pure OA, DDT, OLA, as-produced $\mathrm{AgSbSe}_{2} \mathrm{NCs}\left(\mathrm{AgSbSe}_{2}-\mathrm{OL}\right)$ and $\mathrm{AgSbSe}_{2} \mathrm{NC}$ after ligand removal with $\mathrm{NaNH}_{2}\left(\mathrm{AgSbSe}_{2}-\mathrm{LD}\right)$.

had a metallic lustre, were mechanically robust and had relative densities above 93\% as measured by Archimedes' method. No secondary phases or changes in composition of the $\mathrm{AgSbSe}_{2}$ and $\mathrm{AgSb}_{0.98} \mathrm{Bi}_{0.02} \mathrm{Se}_{2}$ materials during the hot press process were detected by EDX and XRD (Fig. S7 and S8, ESI $\dagger$ ). SEM and TEM characterization showed a very low porosity and large grains, up to several hundred nanometers, during the thermal processes (Fig. 6a and Fig. S9, ESI $\dagger$ ). However, HRTEM characterization allowed discerning crystallographic order domains in the nanometer size regime (Fig. 6b).

Fig. 7 shows the electrical conductivity $(\sigma)$, Seebeck coefficient $(S)$, thermal conductivity $(\kappa)$, and thermoelectric figure of merit $\left(Z T=\sigma S^{2} T / \kappa\right)$ of $\mathrm{AgSbSe}{ }_{2}$ and $\mathrm{AgSb}_{0.98} \mathrm{Bi}_{0.02} \mathrm{Se}_{2}$ pellets. Both materials showed a p-type electronic character. AgSbSe was characterized by a relatively low $\sigma$, which increased from $1380 \mathrm{~S} \mathrm{~m}^{-1}$ at ambient temperature up to $1700 \mathrm{~S} \mathrm{~m}^{-1}$ at $600 \mathrm{~K}$. It showed high Seebeck coefficients reaching up to $462 \mu \mathrm{V} \mathrm{K}^{-1}$ at $695 \mathrm{~K}$, which relates with its flat valence band maximum and multipeak valence band structure. ${ }^{16}$ The introduction of an atomic $0.5 \%$ Bi significantly enhanced the electrical conductivity, which increased from $4520 \mathrm{~S} \mathrm{~m}^{-1}$ at room temperature to $5970 \mathrm{~S} \mathrm{~m}^{-1}$ at $618 \mathrm{~K}$ in $\mathrm{AgSb}_{0.98} \mathrm{Bi}_{0.02} \mathrm{Se}_{2}$. These values are slightly above those previously reported for bulk $\mathrm{AgSb}{ }_{0.98} \mathrm{Bi}_{0.02} \mathrm{Se}_{2}$ produced by solid state methods. ${ }^{16}$ With Bi doping, the Seebeck coefficient decreased to values below $360 \mu \mathrm{V} \mathrm{K} \mathrm{K}^{-1}$, which is consistent with an increase of carrier concentration. Overall, the

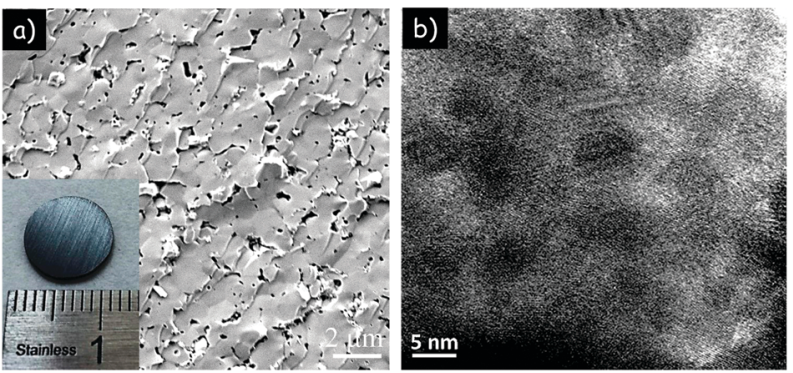

Fig. 6 (a) SEM micrograph of the fractured surface of a AgSbSe 2 pellet (inset); (b) HRTEM micrograph of the same $\mathrm{AgSbSe}_{2}$ pellet showing several crystal nanodomains. 

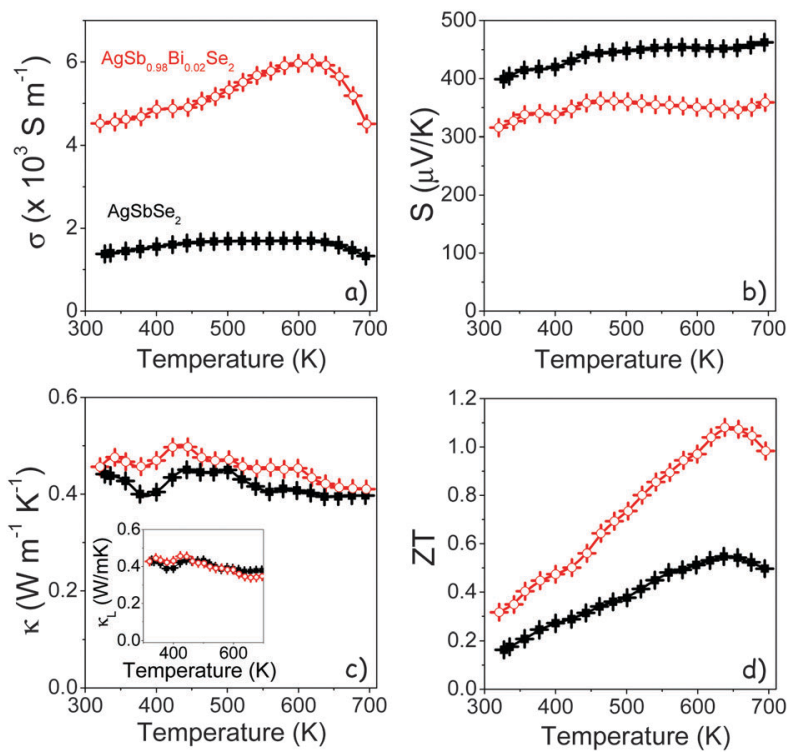

Fig. 7 Temperature dependence of: (a) electric conductivity ( $\sigma$ ); (b) Seebeck coefficient (S); (c) thermal conductivity $(\kappa)$ including lattice thermal conductivity $\left(\kappa_{\mathrm{L}}\right)$ in the inset; and $(\mathrm{d})$ thermoelectric figure of merit $(Z T)$ of $\mathrm{AgSbSe}_{2}(\mathbf{\square})$ and $\mathrm{AgSb}_{0.98} \mathrm{Bi}_{0.02} \mathrm{Se}_{2}(\mathrm{O})$.

$\mathrm{AgSb}_{0.98} \mathrm{Bi}_{0.02} \mathrm{Se}_{2}$ power factor (PF) was higher than that of $\mathrm{AgSbSe}_{2}$, up to $\sim 0.74 \mathrm{~mW} \mathrm{~m}{ }^{-1} \mathrm{~K}^{-2}$ at $580 \mathrm{~K}$ (Fig. S10, ESI $\dagger$ ), and slightly higher than previously reported for bulk $\mathrm{AgSb}_{0.98} \mathrm{Bi}_{0.02} \mathrm{Se}_{2}$ (Fig. S12, ESI $\dagger$ ). ${ }^{16}$

Room temperature Hall measurements provided hole concentrations $p=4 \pm 2 \times 10^{19} \mathrm{~cm}^{-3}$ in $\mathrm{AgSbSe}_{2}$ and 5-fold higher for $\mathrm{AgSb}_{0.98} \mathrm{Bi}_{0.02} \mathrm{Se}_{2}, p=2 \pm 1 \times 10^{20} \mathrm{~cm}^{-3}$. As in related $\mathrm{Cu}_{2} \mathrm{SbSe}_{3}{ }^{42,43}$ and $\mathrm{AgSbTe}{ }_{2}^{44-47}$ compounds, the origin of the p-type conductivity in $\mathrm{AgSbSe}_{2}$ is found in its defect structure and non-stoichiometry, and it is generally associated with Ag vacancies. The introduction of a small amount of an impurity may perturb the AgSbSe ${ }_{2}$ lattice and modify this defect concentration. In the particular case of substituting $\mathrm{Sb}^{3+}$ ions by the larger $\mathrm{Bi}^{3+}$ ions a compressive strain is introduced, which we hypothesize can in part be alleviated by a larger density of $\mathrm{Ag}$ vacancies and thus an increase of the hole concentration. The confirmation of this hypothesis needs in all cases a careful analysis of the defect formation energies within this compound in the presence of $\mathrm{Bi}$, which is out of the scope of the present work. Besides, the relatively low Hall mobilities measured, $2.5 \mathrm{~cm}^{2} \mathrm{~V}^{-1} \mathrm{~s}^{-1}$ and $1.9 \mathrm{~cm}^{2} \mathrm{~V}^{-1} \mathrm{~s}^{-1}$ for $\mathrm{AgSbSe}{ }_{2}$ and $\mathrm{AgSb}_{0.98} \mathrm{Bi}_{0.02} \mathrm{Se}_{2}$, respectively, are consistent with the large degree of disorder, the nanoscale precipitates and the intrinsically large effective mass of holes in this system. The mobility decrease with the introduction of Bi ions is also consistent with the presence of additional point defects scattering charge carriers.

Very low thermal conductivities $\left(\kappa_{\text {total }}=\lambda C_{\mathrm{p}} \rho<0.5 \mathrm{~W} \mathrm{~m}^{-1} \mathrm{~K}^{-1}\right)$ were measured for both $\mathrm{AgSbSe} \mathrm{S}_{2}$ and $\mathrm{AgSb} \mathrm{S}_{0.98} \mathrm{Bi}_{0.02} \mathrm{Se}_{2}$ compounds in the whole studied temperature range of 300 to $700 \mathrm{~K}$ (Fig. 7c). These low thermal conductivities can be explained by an efficient multi-level phonon scattering; that is by nanometer scale precipitates, by the highly disordered lattice and by the strong phonon-phonon interactions from the high degree of anharmonicity of the $\mathrm{Sb}-\mathrm{Se}$ bonds in this material. ${ }^{18}$ In spite of the higher density of defects associated with the presence of $\mathrm{Bi}$, thermal conductivities were slightly higher for $\mathrm{AgSb} \mathrm{Sb}_{0.98} \mathrm{Bi}_{0.02} \mathrm{Se}_{2}$ due to the larger contribution from the electronic thermal conductivity (Fig. 7c inset).

As a result, $Z T$ values of up to 1.1 at $640 \mathrm{~K}$ were obtained for $\mathrm{AgSb}_{0.98} \mathrm{Bi}_{0.02} \mathrm{Se}_{2}$ (Fig. 7d), which represents a two-fold increase over pristine $\mathrm{AgSbSe}$, and is among the best values obtained for a $\mathrm{Pb}$ - and Te-free material at this temperature (Table S1, ESI $\dagger$ ).

\section{Conclusions}

In summary, a scalable colloidal synthesis of monodisperse $\mathrm{AgSbSe}_{2}$ NCs and Bi-doped $\mathrm{AgSb}_{1-x} \mathrm{Bi}_{x} \mathrm{Se}_{2}$ NCs was developed. We showed OLA and OA to be present in dynamic exchange at the surface of the NCs, rendering them soluble in non-polar solvents while allowing kinetic control of their growth. After displacing these surfactants, NCs were used as building blocks to produce $93 \%$ relative density nanomaterials. A preliminary optimization of the doping level resulted in $\mathrm{AgSb}_{0.98} \mathrm{Bi}_{0.02} \mathrm{Se}_{2}$ nanomaterials reaching $Z T=1.1$ at $640 \mathrm{~K}$. This figure of merit is among the largest values reported for a tellurium-free material in the middle temperature range, with the additional advantage of the low-cost and convenient solution processability of the materials and related devices.

\section{Acknowledgements}

This work was supported by the European Regional Development Funds, the Framework 7 program under project UNION (FP7-NMP-2012-310250), the Swiss Federal Commission for Technology and Innovation (CTI, Project Nr. 14698.2 PFIWIW) and the Spanish MINECO project BOOSTER (ENE201346624-C4-3-R). YL thanks the China Scholarship Council (CSC 201406500003) for scholarship support. MI, OD and SO thank AGAUR for their Beatriu de Pinós postdoctoral grant (2013 BPA00344) and PhD grant, respectively. JDR thanks the FWO (Research Foundation Flanders) for financial support. M. V. K. acknowledges partial financial support from the European Union (EU) via FP7 ERC Starting Grant 2012 (Project NANOSOLID, GA No. 306733). The authors also acknowledge the funding from Generalitat de Catalunya 2014 SGR 1638.

\section{Notes and references}

1 K. Wang, C. Steimer, R. Detemple, D. Wamwangi and M. Wuttig, Appl. Phys. A: Mater. Sci. Process., 2005, 81, 1601-1605.

2 H. Soliman, D. Abdel-Hady and E. Ibrahim, J. Phys.: Condens. Matter, 1998, 10, 847.

3 K. Wang, C. Steimer and M. Wuttig, J. Optoelectron. Adv. Mater., 2007, 9, 2008.

4 A. Patel and D. Lakshminarayana, Thin Solid Films, 1982, 98, 59-63. 
5 A. Patel, D. Lakshminarayana and K. Rao, Thin Solid Films, 1982, 94, 51-57.

6 A. Abdelghany, S. Elsayed, D. Abdelwahab, A. A. El Ela and N. Mousa, Mater. Chem. Phys., 1996, 44, 277-280.

7 K. Wojciechowski and M. Schmidt, Phys. Rev. B: Condens. Matter Mater. Phys., 2009, 79, 184202.

8 S. Geller and J. Wernick, Acta Crystallogr., 1959, 12, 46-54.

9 J. Wernick and K. Benson, J. Phys. Chem. Solids, 1957, 3, 157-159.

10 R. Wolfe, J. Wernick and S. Haszko, J. Appl. Phys., 1960, 31, 1959-1964.

11 L. Aggarwal, J. S. Sekhon, S. N. Guin, A. Arora, D. S. Negi, R. Datta, K. Biswas and G. Sheet, Appl. Phys. Lett., 2014, 105, 113903.

12 E. Quarez, K.-F. Hsu, R. Pcionek, N. Frangis, E. Polychroniadis and M. G. Kanatzidis, J. Am. Chem. Soc., 2005, 127, 9177-9190.

13 K. Hoang, S. Mahanti, J. R. Salvador and M. G. Kanatzidis, Phys. Rev. Lett., 2007, 99, 156403.

14 M. Salimi and S. J. Hashemifar, J. Alloys Compd., 2015, 650, 143-148.

15 K. Wojciechowski, J. Tobola, M. Schmidt and R. Zybala, J. Phys. Chem. Solids, 2008, 69, 2748-2755.

16 S. N. Guin, A. Chatterjee, D. S. Negi, R. Datta and K. Biswas, Energy Environ. Sci., 2013, 6, 2603-2608.

17 J. Garza, S. Shaji, A. Rodriguez, T. D. Roy and B. Krishnan, Appl. Surf. Sci., 2011, 257, 10834-10838.

18 T. N. Asokan, K. Urmila, R. Jacob, R. R. Philip, G. Okram, V. Ganesan and B. Pradeep, J. Semicond., 2014, 35, 052001.

19 J. González, S. Shaji, D. Avellaneda, A. Castillo, T. D. Roy and B. Krishnan, Mater. Res. Bull., 2013, 48, 1939-1945.

20 K. Bindu, M. Nair, T. D. Roy and P. Nair, Electrochem. SolidState Lett., 2006, 9, G195-G199.

21 K. Bindu, J. Campos, M. Nair, A. Sanchez and P. Nair, Semicond. Sci. Technol., 2005, 20, 496.

22 K. Wojciechowski, M. Schmidt, J. Tobola, M. Koza, A. Olech and R. Zybała, J. Electron. Mater., 2010, 39, 2053-2058.

23 S. N. Guin, D. S. Negi, R. Datta and K. Biswas, J. Mater. Chem. A, 2014, 2, 4324-4331.

24 D. Li, X. Qin, T. Zou, J. Zhang, B. Ren, C. Song, Y. Liu, L. Wang, H. Xin and J. Li, J. Alloys Compd., 2015, 635, 87-91.

25 S. N. Guin and K. Biswas, J. Mater. Chem. C, 2015, 3, 10415-10421.

26 S. N. Guin, A. Chatterjee and K. Biswas, RSC Adv., 2014, 4, 11811-11815.

27 Z. Liu, J. Shuai, H. Geng, J. Mao, Y. Feng, X. Zhao, X. Meng, R. He, W. Cai and J. Sui, ACS Appl. Mater. Interfaces, 2015, 7, 23047-23055.
28 S. Cai, Z. Liu, J. Sun, R. Li, W. Fei and J. Sui, Dalton Trans., 2015, 44, 1046-1051.

29 E. M. Levin, S. L. Bud'ko and K. Schmidt-Rohr, Adv. Funct. Mater., 2012, 22, 2766-2774.

30 B. A. Cook, M. J. Kramer, J. L. Harringa, M. K. Han, D. Y. Chung and M. G. Kanatzidis, Adv. Funct. Mater., 2009, 19, 1254-1259.

31 D. V. Talapin, J.-S. Lee, M. V. Kovalenko and E. V. Shevchenko, Chem. Rev., 2009, 110, 389-458.

32 M. V. Kovalenko, L. Manna, A. Cabot, Z. Hens, D. V. Talapin, C. R. Kagan, V. I. Klimov, A. L. Rogach, P. Reiss and D. J. Milliron, ACS Nano, 2015, 9, 1012-1057.

33 M. Ibáñez, R. Zamani, S. Gorsse, J. Fan, S. Ortega, D. Cadavid, J. R. Morante, J. Arbiol and A. Cabot, ACS Nano, 2013, 7, 2573-2586.

34 M. Ibáñez, D. Cadavid, U. Anselmi-Tamburini, R. Zamani, S. Gorsse, W. Li, A. M. López, J. R. Morante, J. Arbiol and A. Cabot, J. Mater. Chem. A, 2013, 1, 1421-1426.

35 M. Ibáñez, R. J. Korkosz, Z. Luo, P. Riba, D. Cadavid, S. Ortega, A. Cabot and M. G. Kanatzidis, J. Am. Chem. Soc., 2015, 137, 4046-4049.

36 D. Cadavid, M. Ibáñez, A. Shavel, O. J. Durá, M. L. de la Torre and A. Cabot, J. Mater. Chem. A, 2013, 1, 4864-4870.

37 M. A. Connell, P. J. Bowyer, P. A. Bone, A. L. Davis, A. G. Swanson, M. Nilsson and G. A. Morris, J. Magn. Reson., 2009, 198, 121-131.

38 D. Sinnaeve, Concepts Magn. Reson., Part A, 2012, 40, 39-65. 39 B. Zhou, M. Li, Y. Wu, C. Yang, W. H. Zhang and C. Li, Chem. - Eur. J., 2015, 21, 11143-11151.

40 J. Owen, Science, 2015, 347, 615-616.

41 Z. Hens and J. C. Martins, Chem. Mater., 2013, 25, 1211-1221.

42 X. Li, D. Li, H. Xin, J. Zhang, C. Song and X. Qin, J. Alloys Compd., 2013, 561, 105-108.

43 Y. Zhang, L. Xi, Y. Wang, J. Zhang, P. Zhang and W. Zhang, Comput. Mater. Sci., 2015, 108, 239-249.

44 L.-H. Ye, K. Hoang, A. Freeman, S. Mahanti, J. He, T. M. Tritt and M. Kanatzidis, Phys. Rev. B: Condens. Matter Mater. Phys., 2008, 77, 245203.

45 B. Du, H. Li and X. Tang, J. Alloys Compd., 2011, 509, 2039-2043.

46 V. Jovovic and J. Heremans, Phys. Rev. B: Condens. Matter Mater. Phys., 2008, 77, 245204.

47 R. Mohanraman, R. Sankar, F.-C. Chou, C.-H. Lee, Y. Iizuka, I. P. Muthuselvam and Y.-Y. Chen, APL Mater., 2014, 2, 096114. 